\title{
Simulated Animal Dissection
}

\author{
Cynthia Bruyns ${ }^{1,2}$, Simon Wildermuth ${ }^{1,2}$, Kevin Montgomery ${ }^{1}$ \\ 1. National Biocomputation Center, Stanford - USA \\ 2. Center for Bioinformatics, NASA Ames Research Center - USA \\ \{bruyns, wis, kevin\}@biocomp.stanford.edu
}

\section{Introduction}

We have developed a flexible, multi-user, remote-capable virtual environment system that can be used to simulate rat dissection training. This paper will discuss the technologies required to create a virtual environment for the simulation of a rat dissection procedure incorporating haptic feedback.

\section{Data Acquisition, Segmentation, and Soft-Tissue Deformation}

A multidetector computer tomography was performed under 'in vivo' conditions in two anesthetized rats $(2 \times 0,5 \mathrm{~mm}$ collimation, rotation time $0.8 \mathrm{~s}, \mathrm{FOV} 11 \times 11 \mathrm{~cm}$, $512 \times 512$ matrix). Using iodinated intravenous contrast material allowed the segmentation and reconstruction of the small internal organs, and to create corresponding high resolution polygon surface models. The reconstructed anatomy of the rat is represented within physically- based modeling simulation system. Bones are modeled as rigid objects that are used primarily for constraining the deformable geometry in space. Solution of the deformation equations is done using a localized semi-static solver, which is a simplification that ignores inertial and damping forces but provides a significant increase in performance. In order to speed up the simulation further, we can solve the deformation equations asynchronously, using a multithreaded model on multi-processor Sun (Mountain View, CA) E3500 8x400 $\mathrm{MHz}$ UltraSparc workstation. The simulation system is written in $\mathrm{C}++$ using the OpenGL, GLUT, and POSIX libraries.

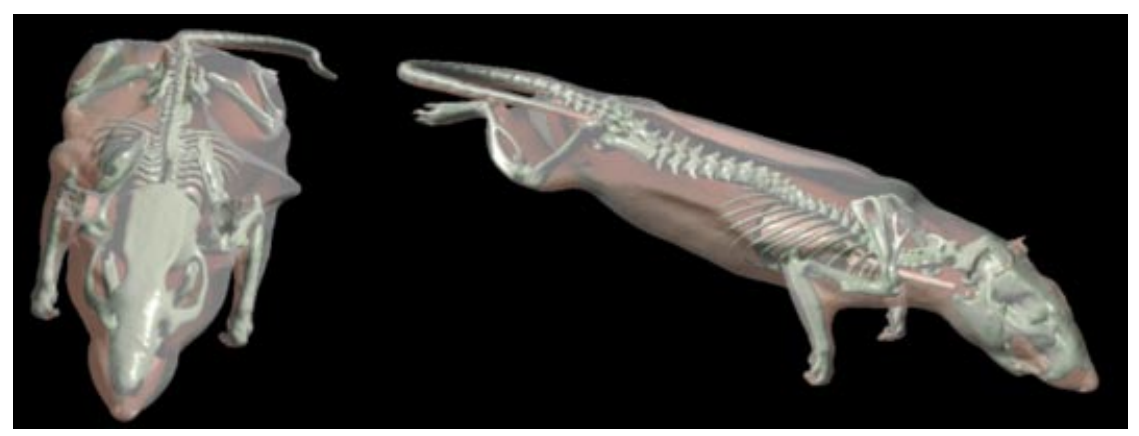

Fig. 1. 3D Surface Model based on high resolution multidetector CT datas

W. Niessen and M. Viergever (Eds.): MICCAI 2001, LNCS 2208, pp. 1345-1346, 2001.

(C) Springer-Verlag Berlin Heidelberg 2001 


\section{Interaction}

In order to allow the user to interact with the environment using actual dissection tools, an Ascension Technologies (Burlington, VT) Flock-of-Birds electromagnetic tracker is attached to real surgical forceps, scalpels and scissors. By mapping the actual three-dimensional position and orientation of the tools to their counterpart in the virtual space, the user can easily interact with the tissue of the virtual rat. Probing the virtual rat can be used to extend the grasping or cutting procedures by adding force-feedback in order to give an impression of the compliance of each tissue. The haptic interface is achieved by a device connected to an embedded processor (Intel Pentium-based dedicated PC) and communicating via 100Mbps Ethernet to the Sun server running the simulation. In this way, we can reduce the computation load on the machine controlling the stability of the haptics device.

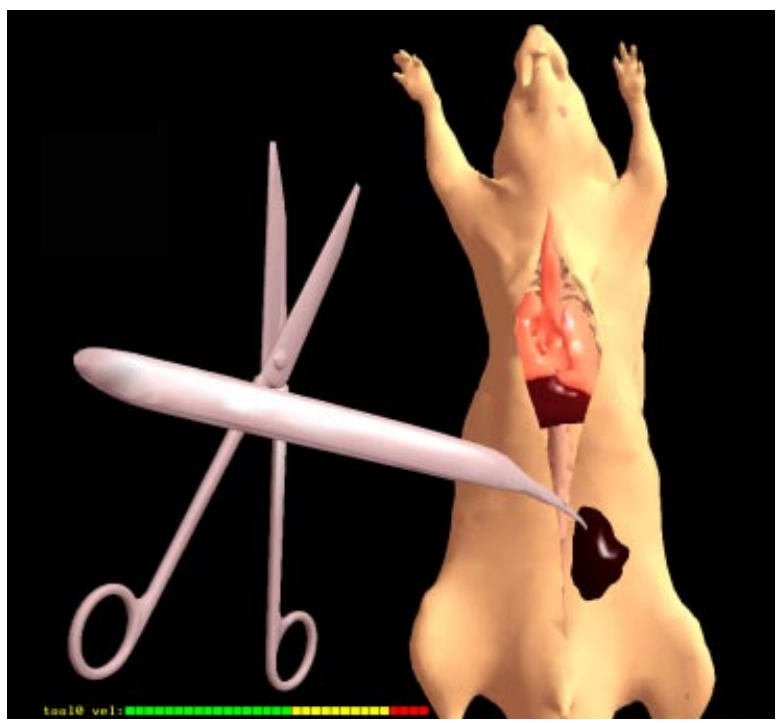

Fig. 2. Demonstrates removing the heart during a virtual animal dissection

\section{Results / Discussion}

We have assembled a preliminary environment for simulating tasks that are performed in animal dissection. This system provides for interaction with both nonforce-feedback and haptic devices and for display with a stereo workstation monitor or tracked head-mounted display. As features are added to the system, there will be an obvious need to evaluate the effectiveness of the environment as a learning tool.

Aknowledgements: This work was supported by grants to the NASA Ames Center for Bioinformatics. Special thanks to Richard Boyle and Marlyn Vasques. 